\title{
An advanced clinician practitioner in arthritis care can improve access to rheumatology care in community-based practice
}

This article was published in the following Dove Press journal: Journal of Multidisciplinary Healthcare

\author{
Vandana Ahluwalia' \\ Tiffany L H Larsen ${ }^{2}$ \\ Carol A Kennedy ${ }^{3}$ \\ Taucha Inrig ${ }^{3}$ \\ Katie Lundon ${ }^{4}$ \\ 'Division of Rheumatology, \\ Department of Internal Medicine, \\ William Osler Health System, \\ Brampton, ON, Canada; ${ }^{2}$ Department \\ of Physiotherapy, Headwaters Health \\ Care Center, Orangeville, ON, \\ Canada; ${ }^{3}$ Musculoskeletal Health and \\ Outcomes Research, St. Michael's \\ Hospital, Toronto, ON, Canada; \\ ${ }^{4}$ Office of Continuing Professional \\ Development and the Department \\ of Medicine, Faculty of Medicine, \\ University of Toronto, ON, Canada
}

Correspondence: Vandana Ahluwalia Division of Rheumatology, Department of Internal Medicine, William Osler Health System, 3/4-40 Finchgate Boulevard, Brampton, ON, Canada

Tel + I 905799 I850; Ext 22I

$\mathrm{Fax}+\mathrm{I} 9057998040$

Email vandana@sympatico.ca
Objective: To facilitate access and improve wait times to a rheumatologist's consultation, this study aimed to 1) determine the ability of an advanced clinician practitioner in arthritis care (ACPAC)-trained extended role practitioner (ERP) to triage patients with suspected inflammatory arthritis (IA) for priority assessment by a rheumatologist and 2) determine the impact of an ERP on access-to-care as measured by time-to-rheumatologist-assessment and time-to-treatment-decision.

Materials and methods: A community-based ACPAC-trained ERP triaged new referrals for suspected IA. Patients with suspected IA were booked to see the rheumatologist on a priority basis. Diagnostic accuracy of the ERP to correctly identify priority patients; the level of agreement between ERP and rheumatologist (Kappa coefficient and percent agreement); and the time-to-treatment-decision for confirmed cases of IA were investigated. Retrospective chart review then compared time-to-rheumatologist-assessment and time-to-treatment-decision in the solo-rheumatologist versus the ERP-triage model.

Results: One hundred twenty-one patients were triaged. The ERP designated 54 patients for priority assessment. The rheumatologist confirmed IA in 49/54 (90.7\% positive predictive value [PPV]). Of the 121 patients, 67 patients were designated as nonpriority by the ERP, and none were determined to have IA by the rheumatologist (100\% negative predictive value [NPV]). Excellent agreement was found between the ERP and the rheumatologist (Kappa coefficient 0.92, 95\% CI: 0.84-0.99). In the ERP-triage model, time-from-referral-to-treatment-decision for patients with IA was 73.7 days (SD 40.4, range 12-183) compared with 124.6 days (SD 61.7, range 26-359) in the solo-rheumatologist model (40\% reduction in time-to-treatment-decision). Conclusion: A well-trained and experienced ERP can shorten the time-to-Rheumatologistassessment and time-to-treatment-decision for patients with suspected IA.

Keywords: rheumatology, health services accessibility, interprofessional relations, community health services, integrated delivery systems

\section{Introduction}

Rapid access to rheumatology care has become increasingly important as early aggressive treatment with disease-modifying antirheumatic drugs (DMARDs) and/or biologic agents can prevent irreversible joint damage and long-term disability in patients with IA. Early intervention with a treatment decision within 6 weeks of referral in patients with suspected $\mathrm{RA}^{1}$ is directly related to improved clinical outcomes, functional status, and a higher quality of life..$^{2-6}$ In Ontario, only $38 \%$ of patients with RA are currently meeting this benchmark for first point of contact with a rheumatologist. ${ }^{7}$ Fewer than $50 \%$ receive a DMARD within 6 months of symptom onset. ${ }^{8}$ 
In Canada, more than 272,000 people currently live with RA, representing $0.9 \%$ of the Canadian adult population, and this number is expected to increase by $1.3 \%$ in the next 30 years. ${ }^{1}$ This increased prevalence of patients with IA will be exacerbated by a concurrent shortage of practicing rheumatology specialists ${ }^{9}$ and a progressive deficit in their numbers within the next 10 years across Canada has been reported..$^{10}$ The potential deficiencies in rheumatology care are further exacerbated by access barriers to rheumatology services, particularly for populations with low access to primary care physicians or more vulnerable populations. ${ }^{11}$ These studies underscore the urgent need to address shortages in traditional human health resources and thus consider more efficient and effective models to deliver arthritis care. In order to meet these service-demand issues in rheumatology, one viable option under consideration is the adoption of alternative models of care to increase capacity, not only in highly populated urban areas, but also in remote and rural areas. At the core of these new models of arthritis care is the reliance upon strong inter-professional collaborative relationships between existing rheumatologists and other highly trained, competent health care professionals. In such service delivery models, the skillsets of advanced practice or extended role practitioners (ERPs) are leveraged and with medical directives or authorized activities in place, ${ }^{12}$ have significant potential to magnify the workforce capacity and improve access to care for patients living with arthritis. ${ }^{13-15}$ The impetus to facilitate access and shorten wait times to a rheumatologist's assessment has gained traction elsewhere and includes consideration of how other health professionals, with appropriate training, might be able to fulfill certain roles as "gatekeepers" ${ }^{16}$ In Canada, it has been similarly proposed that by using a broader range of providers in the centralized intake of patients with rheumatoid and osteoarthritic conditions, there is the potential to improve access to care and ultimately enhance the patient care experience. ${ }^{17}$

The development of specialized training programs to educate and accredit ERPs in arthritis care is central to preparing the human health resource for advanced practice. ${ }^{18-20}$ The University of Toronto-based ACPAC ${ }^{21}$ program was developed specifically to promote the extension of roles of experienced arthritis healthcare professionals (including Physical Therapists, Occupational Therapists, and Nurses) to work as ERPs in two identified streams of arthritis care: triage and ongoing clinical care. Graduates of the ACPAC program ${ }^{21}$ represent a new cadre of practitioners, having received additional training at the postlicensure level and undergone formal evaluation to establish competency in advanced assessment, diagnosis, and management of select arthritic conditions. ${ }^{18}$ Their roles have been evaluated and described in a global construct at the health services level, with favourable outcomes for the patient ${ }^{15}$ as well as at clinical utilization and early system integration and change levels. ${ }^{13,14,19,22-24}$

While there are many described roles of advanced practice physiotherapists in rheumatology practice, there is a lack of knowledge around their specific management of patients with suspected IA. ${ }^{25}$ To date, the contextual efficacy of ERPs working within a shared-care model in a community-based rheumatology practice has not been studied. The primary objective of this study was to determine the ability of an experienced ACPAC program-trained ERP working in a triage role to correctly identify patients with suspected IA for priority assessment by a rheumatologist. A secondary objective was to determine the impact of an ACPAC program-trained ERP on access-to-care for patients with IA, as measured by time-torheumatologist-assessment and time-to-treatment-decision.

\section{Materials and methods}

A prospective study design, integrated into routine clinical practice was used to evaluate the ability of the ACPAC-trained ERP to identify priority (suspected IA) patients using a standardized electronic medical record (EMR) triage form (Figure 1) and assess the impact of the ERP-triage model on time-to-rheumatologist-assessment and time-to-treatmentdecision. A retrospective chart review was then conducted to compare time-to-rheumatologist-assessment and timeto-treatment-decision in the solo-rheumatologist practice in Brampton, ON, Canada. The ERP had 13 years of clinical experience as a physical therapist and 7 years of clinical experience as an ACPAC ERP. The rheumatologist had 18 years of clinical experience in a community practice setting.

\section{Study population}

Consecutive adult patients ( $>18$ years of age) newly referred by local primary care physicians between January 2012 and March 2013 were paper triaged by the rheumatologist to see the ERP if there was a suspicion of IA ("gray zone" patients). Gray zone patient referrals included those with some features suggestive of IA either in the form of laboratory or radiographic investigations and were selected for the shared-care model. Excluded were patients who did not need triaging: referrals that had adequate documentation to support a diagnosis of IA (swollen joints, positive serology, elevated inflammatory markers, other features of a connective tissue disease, or radiologic evidence of IA), and those who clearly did not have IA (OA, crystal diseases, FM, and other local musculoskeletal [MSK] conditions). Also excluded were 
Dear Dr:

CC:
Patient's name:

DOB:

Assessment date:
Consent for assessment:

HPI:

\section{Physical examination:}

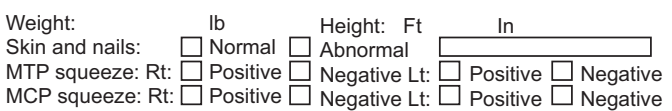

MCP squeeze: Rt: $\square$ Positive $\square$ Negative Lt: $\square$ Positive $\square$ Negative
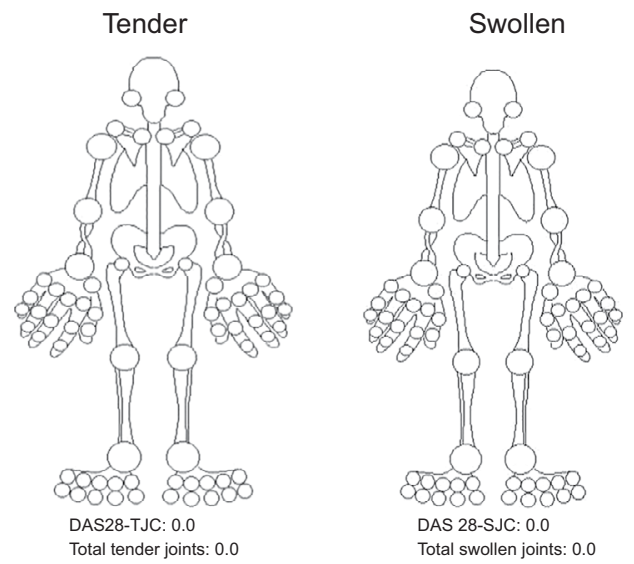

Impression (ERP query diagnosis)

$\square$ Seropositive

$\square$ Seronegative

$\square$ MSK

$\square$ Fibromyalgia

Plan

$\square$ B/W ordered

X-ray ordered

$\square$ Referral Arthritis Society

$\square$ Booked priority

$\square$ Booked nonpriority

$\square$ Booked injection clinic
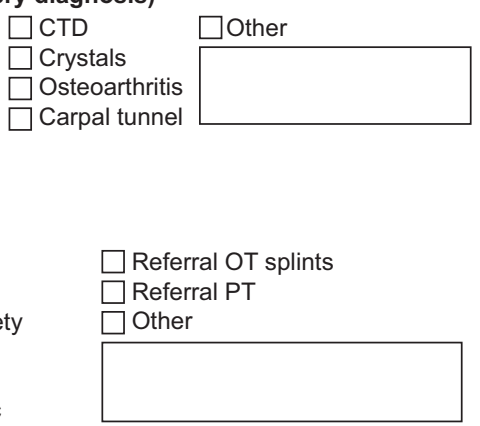

Figure I EMR triage form.

Abbreviations: EMR, electronic medical record; ERP, extended role practitioner; CT, computed tomography; CTD, mixed connective tissue disease; MSK, musculoskeletal (conditions); OT, occupational therapy; PT, physical therapy; CBC, complete blood count; RF, rheumatoid factor; ANA, antinuclear antibodies; ESR, erythrocyte sedimentation rate; HPI, history of presenting illness; MTP, metatarsophalangeal; MCP, metacarpophalangeal; CRP, C-reactive protein.

patients who had been seen by a rheumatologist within the past 5 years, or who needed a second opinion for a previously diagnosed rheumatic condition. The excluded patients were seen directly by the rheumatologist.

\section{Triage intervention}

Prior to the triage assessment by the ACPAC ERP, patients completed a medical history form containing demographics, past medical history, medications, allergies, social history, and family history as per usual care. The ACPAC ERP's triage assessment consisted of a timed 15-minute appointment with findings documented on a standardized EMR form (Figure 1).

Measures collected as part of the standardized EMR assessment form included a history of the current condition, patient-reported pain, morning stiffness, fatigue, sleep, and a patient global assessment of health. Laboratory data and imaging reports provided by the primary care physician in the referral were reviewed. A physical exam that included blood pressure measurement, grip strength, tender joint count, swollen joint count, enthesial, and tendon involve- 
ment as well as a spinal exam was documented. Suspected diagnoses were considered and further investigations were ordered as needed. The ERP had advanced medical directives to order additional laboratory tests or X-rays as necessary by clinical judgment, with the results forwarded to the rheumatologist. The ERP did not have access to the laboratory and X-ray results, but relied solely upon clinical judgment to prioritize patients with suspected IA (including RA, PsA, AS, SLE, CTD, and other IA) to see the rheumatologist. Patients in whom IA was not suspected (including OA, crystal, FM and other MSK conditions) were given education, exercises, joint protection advice, referral for splinting as needed, and booked for a rheumatologist's appointment on a non-priority basis. The rheumatologist independently assessed both the priority and non-priority patients, and the diagnosis and treatment recommendations were documented in a typed consultation note. At the time of consultation, the rheumatologist had access to the ERP's EMR triage assessment and additional test results as ordered by the ERP. The rheumatologist's final diagnosis was based on the patient's history, physical examination, and investigations sent by the referring physician and/or ordered by the ERP.

A comparative group was derived from a retrospective chart review conducted over a 25 -month period on a similar number of patients diagnosed with IA assessed under the solo-rheumatologist model. Time-to-rheumatologist-assessment and time-to-treatment-decision were calculated for both the triage intervention and comparative groups.

\section{Analysis}

Data were entered into an Excel spreadsheet, and all analyses were conducted using Excel and SAS version 9.4. ${ }^{26}$ Descriptive statistics were used to report patient demographics of the sample. As this was a quality improvement initiative, no power calculation was performed.

Diagnostic accuracy of the priority rating by the ERP and descriptive statistics were performed. Sensitivity (Se) (Se $=$ true positive $(\mathrm{A}) /(\mathrm{A}+$ false negative $(\mathrm{C}))$ was considered to be the proportion of patients with a positive criterion test that the ERP correctly detected (true positive rate); Specificity $(\mathrm{Sp})(\mathrm{Sp}=$ true negative $(\mathrm{D}) /$ false positive $(\mathrm{B})+$ (D) was the proportion of patients with a negative criterion test that the ERP correctly detected (true negative rate); positive predictive value $(\mathrm{PPV})(\mathrm{PPV}=\mathrm{A} / \mathrm{A}+\mathrm{B})$ was the probability that a patient with a positive triage intervention test actually had a positive criterion test; negative predictive value $(\mathrm{NPV})(\mathrm{NPV}=\mathrm{D} / \mathrm{C}+\mathrm{D})$ was the probability that a patient with a negative triage intervention test actually had a negative criterion test.
Kappa coefficient was used to assess the level of agreement between the ACPAC ERP priority rating and the rheumatologist's diagnosis of IA. Percentage (or observed) agreement (proportion of cases for which the ERP and rheumatologist agreed) was also calculated.

Wait times were measured as the number of days from primary care referral to rheumatologist assessment (time-to-rheumatologist-assessment) and the number of days to the treatment decision (time-to-treatment-decision) in both the ERP-triage model and the retrospective review of the solo-rheumatologist model for patients diagnosed with IA.

\section{Ethical review}

Permission to perform the retrospective chart review and use of routinely collected data from those patients included in this study was approved by the REB of the William Osler Health System. Individual signed informed consent was not required by the REB for quality assurance retrospective review of charts. All patient data were maintained with confidentiality in accordance with the Declaration of Helsinki.

\section{Results \\ Study population}

One hundred forty-two "gray zone" patients were seen and triaged by the ERP; however, 21/142 (14.8\%) canceled or did not show up for the rheumatologist's assessment leaving 121 in the study population. Of those with suspected IA who did not attend the appointment with the rheumatologist $(n=6), 100 \%$ were female, and the mean age (39.8 years) of this group was also younger than other groups (Figure 2). Ultimately $49 / 121$ (40\% of study sample) were confirmed to have IA by the rheumatologist's assessment. The ERP's allocation of patients to suspected diagnostic subcategories for both IA and non-IA conditions was also very similar to those confirmed by the rheumatologist (Figure 3 ).

\section{Triage intervention}

True positives: Of the 49 patients who were assessed by the rheumatologist as having a diagnosis of IA, the ERP correctly identified 49 (100\%) as suspected IA.

True negatives: Of the 72 patients who were assessed by the rheumatologist as having a non-IA diagnosis, the ERP correctly identified 67 (Sp 93\%) as suspected non-IA.

False positives: Of the 72 patients who were assessed by the rheumatologist as having a non-IA diagnosis, the ERP considered 5 (6.9\%) as suspected IA.

False negatives: Of the 49 patients who were assessed by the rheumatologist as having a diagnosis of IA, the ERP considered none (Se 100\%) as suspected non-IA. 


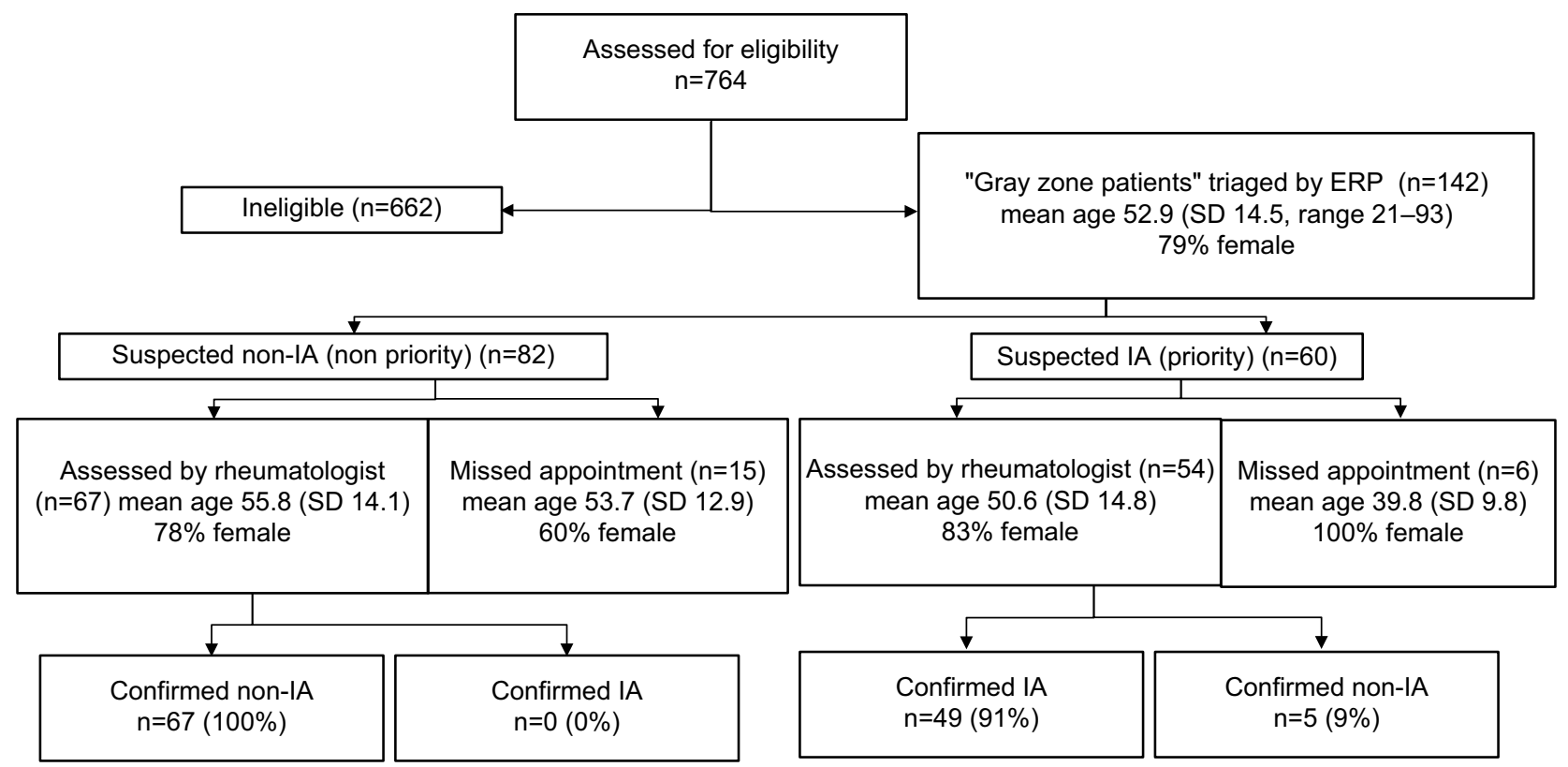

Figure 2 Patient allocation and demographics.

Abbreviations: ERP, extended role practitioner; IA, inflammatory arthritis.

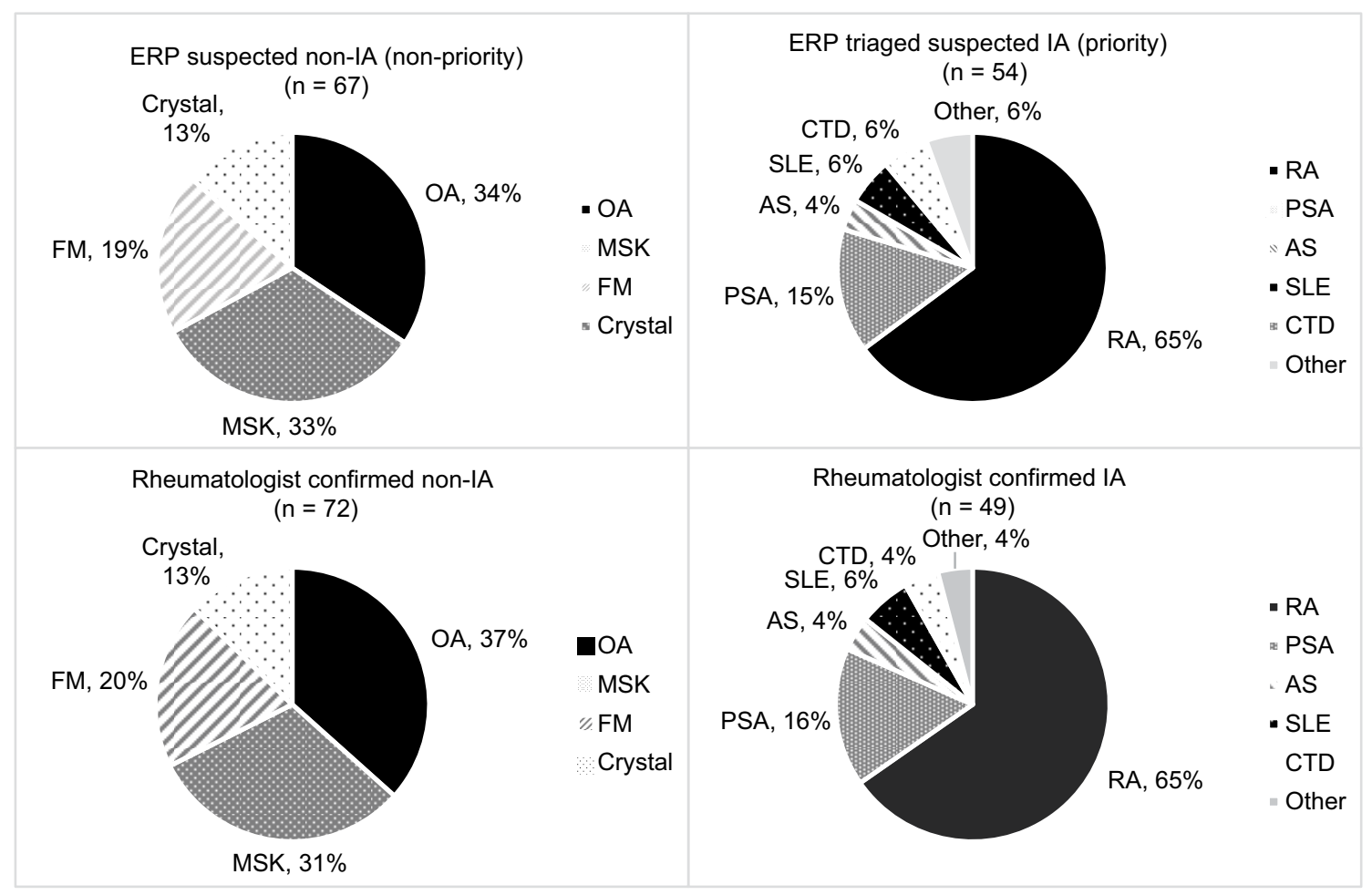

Figure 3 ERP-suspected vs Rheumatologist-confirmed diagnoses.

Abbreviations: ERP, extended role practitioner; CTD, mixed connective tissue disease; AS, ankylosing spondylitis; PSA, psoriatic arthritis; SLE, systemic lupus erythematosus; RA, rheumatoid arthritis; FM, fibromyalgia; MSK, musculoskeletal (conditions); OA, osteoarthritis.

The PPV for the ERP (ie, cases where the patient was triaged as suspected IA) and the rheumatologist diagnosed the patient with IA was $90.7 \%$ (95\% CI: $79.7 \%-96.9 \%$ ). The NPV for the ERP (ie, cases where the patient was triaged as suspected non-IA and the rheumatologist diagnosed the patient as non-IA) was $100 \%$ (95\% CI: 94.6\%-100\%). The Kappa coefficient between the ERP priority rating and the rheumatologist diagnosis was 0.92 (95\% CI: 0.84-0.99), where 1.0 represents perfect agreement (Table 1). 
Table I Comparison between triage intervention (ERP) and criterion (rheumatologist)

\begin{tabular}{|c|c|c|c|c|c|}
\hline & & \multicolumn{2}{|c|}{ Criterion=rheumatologist } & \multirow[t]{2}{*}{ Total } & \\
\hline & & Diagnosed IA & Diagnosed non-IA & & \\
\hline \multirow{6}{*}{ 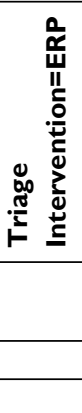 } & Priority (Suspected IA) & $\begin{array}{l}\mathrm{n}=49 \\
\text { True Positive (A) }\end{array}$ & $\begin{array}{l}\mathrm{n}=5 \\
\text { False Positive (B) }\end{array}$ & $\begin{array}{l}n=54 \\
A+B\end{array}$ & $\begin{array}{l}\text { PPV }=90.7 \% \\
\text { (Cl 95\%: 79.7\%-96.9\%) }\end{array}$ \\
\hline & $\begin{array}{l}\text { Nonpriority (Suspected } \\
\text { Non-IA) }\end{array}$ & $\begin{array}{l}\mathrm{n}=0 \\
\text { False Negative }(\mathrm{C})\end{array}$ & \begin{tabular}{|l|}
$n=67$ \\
True Negative (D)
\end{tabular} & $\begin{array}{l}n=67 \\
C+D\end{array}$ & $\begin{array}{l}N P V=100 \% \\
(C l 95 \%: 94.6 \%-100 \%)\end{array}$ \\
\hline & Total & $\begin{array}{l}n=49 \\
A+C\end{array}$ & $\begin{array}{l}n=72 \\
B+D\end{array}$ & $\begin{array}{l}n=|2| \\
A+B+C+D\end{array}$ & \\
\hline & & $\begin{array}{l}\mathrm{Se}=100 \% \\
(\mathrm{Cl} 95 \%: 92.5-100 \%)\end{array}$ & $\begin{array}{l}\mathrm{Sp}=93.1 \% \\
(\mathrm{Cl} 95 \%: 84.5-97.7 \%)\end{array}$ & & \\
\hline & Percent agreement & ||$|6 /| 2 \mid(95.9 \%)$ & & & \\
\hline & Kappa & 0.92 (Cl 95\%: 0.84-0.99) & & & \\
\hline
\end{tabular}

Abbreviations: ACPAC, Advanced Clinician Practitioner in Arthritis Care; ERP, extended role practitioner; IA, inflammatory arthritis; NPV, negative predictive value; PPV, positive predictive value; Se, sensitivity; Sp, specificity.

\section{Comparative wait times: time-to- rheumatologist assessment and time-to- treatment-decision}

The average number of days from primary care referral to the solo-rheumatologist assessment and initiation of treatment was determined for those patients confirmed to have a diagnosis of IA and compared in the ERP triage versus the solorheumatologist model by a retrospective chart review. One hundred and ten $(\mathrm{n}=110)$ charts were reviewed on patients with suspected IA in the solo-rheumatologist model, where a similar distribution of patients diagnosed with IA (48\%) and with non-IA (52\%) was found. The ERP-triage model mean time-to-rheumatologist-assessment for patients with suspected IA was 81.6 days (SD 44.2, range 11-210) compared with 77.6 days (SD 41.5, range 8-168) in the solo-rheumatologist model representing a slight (4 day) increase in time-to-rheumatolgistassessment. The priority patients waited an average of 42.7 days from referral to ERP assessment and 38.9 days from ERP assessment to rheumatologist-assessment. The ERPtriage model mean time-to-treatment-decision for patients with suspected IA was 73.7 days (SD 40.4, range 12-183) compared with 124.6 days (SD 61.7, range 26-359) in the solo-rheumatologist model representing a $40 \%$ reduction in time-to-treatment-decision (Figure 4).

Gray zone patients deemed non-priority (suspected nonIA) in the ERP-triage model waited an average of 115.6 days to rheumatologist assessment. These non-priority patients waited an average of 48.7 days from referral to ERP triage and 66.9 days from ERP to rheumatologist assessment.

\section{Discussion}

In this study, we evaluated the ability of an ACPAC-trained ERP to correctly identify patients with suspected IA, the agreement with the rheumatologist's subsequent diagnosis, and the impact the ERP-triage model had on time-to-rheumatologist-assessment and time-to-treatment-decision. The ERP was able to correctly identify suspected IA patients for priority assessment by the rheumatologist $(\mathrm{PPV}=90.7 \%)$. There was excellent agreement (Kappa coefficient $=0.92)$ in diagnostic accuracy between the rheumatologist and the ERP. This model resulted in considerable wait-time reduction for treatment-decision for patients with suspected IA and also allowed earlier nonmedical therapy to be initiated for suspected non-IA patients. Furthermore, when the diagnostic agreement between the ACPAC ERP and the rheumatologist was probed, there was close alignment of how patients were assigned within the diagnostic subcategories of IA and nonIA conditions.

In this sample population, $60 \%$ of patients referred for rheumatology consult did not have inflammatory disease requiring urgent consultation. Similarly, in an orthopedic setting, at least $70 \%$ of patients referred to orthopedic surgeons did not require surgical management ${ }^{27,28}$ with strong agreement in making that decision observed between highly trained advanced practice physical therapists and orthopedic surgeons. ${ }^{24,27,29}$ Forty percent of the patients triaged by the ACPAC-trained ERP in this sample across a 14-month period presented with urgent inflammatory conditions allowing their care to be prioritized to the rheumatologist. Of further note, the ERP had advanced directives to order additional laboratory or radiological investigations at the time of the triage assessment. When these patients presented to the rheumatologist for confirmatory assessment, the results of those tests were available, thus eliminating the lag between initial assessment and treatment-decision found in the solorheumatologist model. The time-to-treatment-decision is 
Time-to-rheumatologist-assessment for priority patients with IA

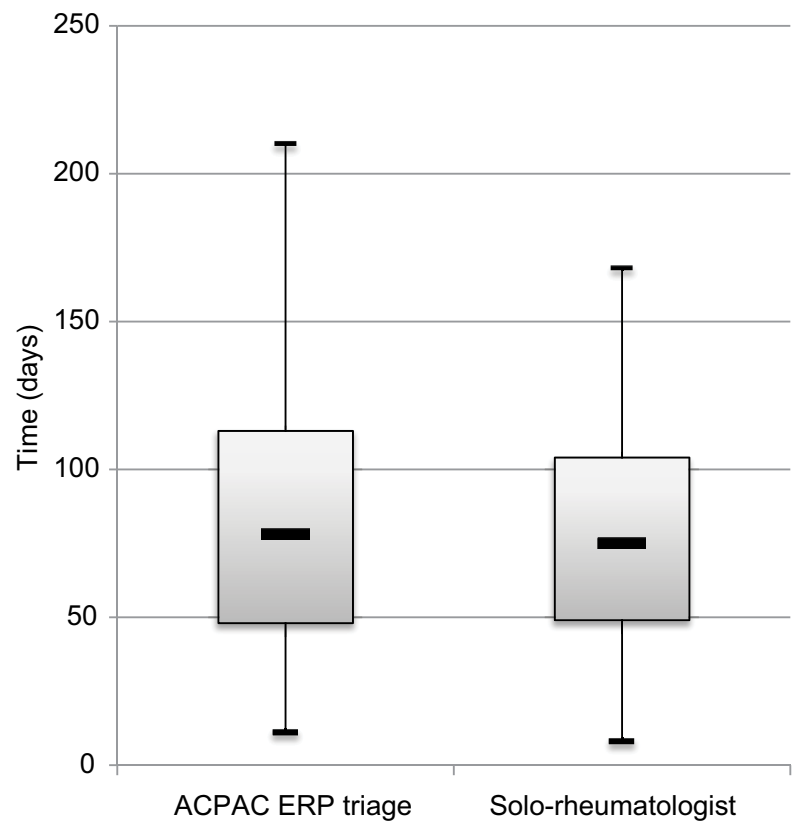

Time-to-treatment-decision for priority patients with IA

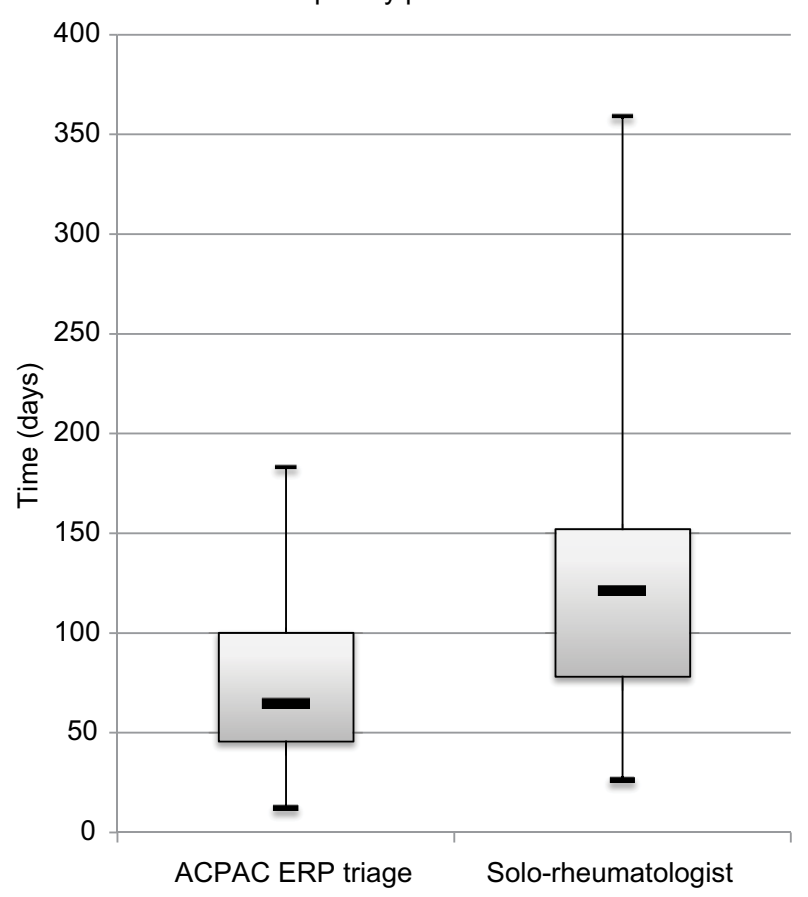

Figure 4 Comparative time-to-assessment/treatment decision for priority patients with IA in the ERP-triage vs Solo-rheumatologist models of care. Abbreviations: ACPAC, Advanced Clinician Practitioner in Arthritis Care; IA, inflammatory arthritis; ERP, extended role practitioner.

the outcome that is important clinically. Initial consultation with the rheumatologist does not ascertain the initiation of an intervention; however, having the results of ERP-initiated investigations available at the time of the rheumatologist consultation allowed the physician to make a treatment decision at the first visit in this shared-care model.

If a patient was deemed priority by the ERP, they were booked on best effort within 4-6 weeks to see the rheumatologist. If a patient was deemed non-priority by the ERP, the patient was booked in $\sim 3-4$ months to see the rheumatologist. It is typical of patients with non-IA in this solo-rheumatologist's practice to be seen 6-9 months after referral. Gray zone patients with suspected non-IA in the ERP-triage model were seen earlier by the rheumatologist as they had the benefit of additional laboratory or radiologic investigations and also had the benefit of being supported with conservative management strategies given by the ERP prior to the first rheumatologist visit.

Six young women (mean age 39.8 years) in whom the ACPAC ERP suspected having IA did not follow through with their priority appointment with the rheumatologist. As per routine clinic practice, attempts to contact these patients and their referring physicians were made. In comparison, there is typically a $\sim 10 \%$ no show rate for new patients with suspected IA in this community rheumatology practice. In other populations of women in this age profile afflicted by a significant medical condition, similarly poor attention to health care has been noted. As a possible explanation, the lack of attention to personal health needs has been reported among those in caregiving roles such as might be experienced in child-bearing or rearing years. ${ }^{30}$

\section{Strengths and limitations}

A limitation of the study may be that the rheumatologist was neither blinded to the original paper triage of the patient nor to the ERP's suspected diagnosis and was able to review the ERP's assessment during the initial rheumatologist visit. Patients were neither randomly assigned to the intervention nor was a power calculation performed to determine sample size. This study was conducted as part of our practice's quality improvement and not as a clinical trial, therefore it was not designed to assess interrater reliability. However, this represents the reality of a clinical setting, and it would not be ethical to withhold test results from the rheumatologist for review at the time of patient consultation.

Another limitation is that this study compared triage priority ratings made by one expert ERP and as such, the findings may not be generalizable to other settings. The 
familiarity of the ERP and the rheumatologist with the notion of shared-care is a strength of our clinical model. The ACPAC ERP had trained and worked with the rheumatologist for $>7$ years and was aware of the rheumatologist's practice patterns which may have influenced the outcome of this study.

In another shared-care ERP model, patients with suspected non-IA were shown to experience slightly longer wait times to see a rheumatologist than they would have experienced in a solo-rheumatologist model of care, ${ }^{23}$ but they were not dissatisfied with this outcome. ${ }^{31}$ In the present study, gray zone suspected non-IA patients were seen earlier (3-4 months) than the traditional solo-rheumatology model (6-9 months). This ERP shared-care model additionally allowed investigations, non-pharmacologic treatment modalities, health education, and self-management strategies as permitted by the medical directives to be given to these patients during the time between the ERP triage assessment and the rheumatologist's assessment. The subject of patient satisfaction with both solorheumatologist and ERP models of care is important and will be explored in future research conducted by our team.

A strength of the ERP-triage model was the clinically significant reduction in time-to-treatment-decision for those patients with suspected IA. Early treatment reduces patient suffering, reduces joint damage, improves mobility and function, and also has significant fiscal implications to patients, their families, and to society. ${ }^{1-7,32,33}$

\section{Conclusion}

Embedding an ERP-triage model into community rheumatology care can be ideal in settings where there is a shortage of human health resources for arthritis care. The ability of an experienced and well-trained ERP to assess patients with suspected IA resulted in prompt rheumatology consultation and earlier treatment decisions. A formal postlicensure training opportunity such as the ACPAC program ensures acquisition of the advanced skills and knowledge necessary to support the development of extended practice roles in appropriately chosen health care providers already invested in arthritis care.

The triage role of the ERP outlined in this paper is only one of the potential roles that can be supported in the sharedcare model. An ACPAC ERP is trained to work independently seeing patients for whom DMARDs/ biologics have been initiated and for interim follow-up visits to assess for compliance and side effects as well as early response to treatment. These practitioners could carry out an IA care plan consisting of patient education on their disease and treatment to improve adherence, comorbidity identification, and ensuring vaccinations are up to date. The ERP could also work in parallel stream alongside the rheumatologist seeing stable patients in follow-up and identifying those patients who have experienced a flare and thus require a rheumatologist's prompt assessment. The study of further potential use of ACPAC ERPs to improve access to health care includes evaluating their deployment in primary care, for example, family health teams, follow-up, and monitoring of patients with IA, etc. These areas need further development and research at the policy and health service delivery level to ensure that the ACPAC ERP is most effectively positioned and enabled to improve access to care so that the most urgent patients receive prompt rheumatology assessment and treatment.

\section{Abbreviations}

ACPAC, Advanced Clinician Practitioner in Arthritis Care; APP, advanced practice physiotherapists; AS, ankylosing spondylitis; CTD, mixed connective tissue disease; DMARDs, disease-modifying antirheumatic drugs; EMR, electronic medical record; ERP, extended role practitioner; FM, fibromyalgia; IA, inflammatory arthritis; MSK, musculoskeletal (conditions); NPV, negative predictive value; OA, osteoarthritis; PPV, positive predictive value; PsA, psoriatic arthritis; RA, rheumatoid arthritis; REB, Research Ethics Board; Se, sensitivity; SLE, systemic lupus erythematosus; Sp, specificity.

\section{Acknowledgments}

The authors would like to acknowledge the assistance of Monish Ahluwalia. Financial support was received from the Bristol Myers Squibb Unrestricted Educational Grant. The abstract of this paper was presented at the 69th Annual Meeting of the Canadian Rheumatology Association (2014) as a poster presentation with interim findings. The poster's abstract was published under "Poster Presentations" in The Journal of Rheumatology 2014; 41:7 doi:10.3899/ jrheum.140420. Additionally, the abstract of this paper was presented at the 2014 American College of Rheumatology Meeting as a poster presentation with interim findings. The poster's abstract was published under "ACR/ARHP Abstract Supplement" in Arthritis and Rheumatology 2014, 66:10 (supplement): https://doi.org/10.1002/art.38914.

\section{Disclosure}

The authors report no conflicts of interest in this work.

\section{References}

1. Arthritis Alliance of Canada. A Pan-Canadian Approach to Inflammatory Arthritis Models of Care, 30 April 2014. Canada: Arthritis Alliance of Canada; 2014.

2. Breedveld $F$. The value of early intervention in RA - a window of opportunity. Clin Rheumatol. 2011;30(Suppl 1):33-39. 
3. Bykerk V, Emery P. Delay in receiving rheumatology care leads to long-term harm. Arthritis Rheum. 2010;62(12):3519-3521.

4. Ozminkowski RJ, Burton WN, Goetzel RZ, Maclean R, Wang S. The impact of rheumatoid arthritis on medical expenditures, absenteeism, and short-term disability benefits. J Occup Environ Med. 2006;48(2):135-148

5. Jamal S, Alibhai SM, Badley EM, Bombardier C. Time to treatment for new patients with rheumatoid arthritis in a major metropolitan city. J Rheumatol. 2011;38(7):1282-1288.

6. Villeneuve E, Nam JL, Bell MJ, et al. A systematic literature review of strategies promoting early referral and reducing delays in the diagnosis and management of inflammatory arthritis. Ann Rheum Dis. 2013;72(1):13-22.

7. Widdifield J, Bernatsky S, Bombardier C, Paterson M. Rheumatoid arthritis surveillance in Ontario: monitoring the burden, quality of care and patient outcomes through linkage of administrative health data. Healthc Q. 2015;18(3):7-10.

8. Tavares R, Pope JE, Tremblay JL, et al. Time to disease-modifying antirheumatic drug treatment in rheumatoid arthritis and its predictors a national, multicenter, retrospective cohort. J Rheumatol. 2012;39(11): 2088-2097.

9. Widdifield J, Paterson JM, Bernatsky S, et al. The rising burden of rheumatoid arthritis surpasses rheumatology supply in Ontario. Can J Public Health. 2013;104(7):450-455.

10. Barber CE, Jewett L, Badley EM, et al. Stand up and be counted: measuring and mapping the rheumatology workforce in Canada. J Rheumatol. 2016.

11. Badley EM, Canizares M, Gunz AC, Davis AM. Visits to rheumatologists for arthritis: the role of access to primary care physicians, geographic availability of rheumatologists, and socioeconomic status. Arthritis Care Res. 2015;67(2):230-239.

12. Federation of Health Regulatory Colleges of Ontario. An Interprofessional Guide on the Use of Orders, Directives and Delegation for Regulated Health Professionals in Ontario; 2017. Available from: http:// www.regulatedhealthprofessions.on.ca/md-guide-home.html. Accessed January 4, 2017.

13. Passalent LA, Kennedy C, Warmington K, et al. System integration and clinical utilization of the Advanced Clinician Practitioner in Arthritis Care (ACPAC) program-trained extended role practitioners in Ontario: a two-year, system-level evaluation. Healthc Policy. 2013;8(4): 56-70.

14. Lineker SC, Lundon K, Shupak R, Schneider R, Mackay C, Varatharasan $\mathrm{N}$. Arthritis extended-role practitioners: impact on community practice (an exploratory study). Physiother Can. 2011;63(4):434-442.

15. Warmington K, Kennedy CA, Lundon K, et al. The patient perspective: arthritis care provided by Advanced Clinician Practitioner in Arthritis Care program-trained clinicians. Open Access Rheumatol. 2015;7:45-53.

16. Gärtner M, Fabrizii JP, Koban E, et al. Immediate access rheumatology clinic: efficiency and outcomes. Ann Rheum Dis. 2012;71(3): 363-368.

17. Suter E, Birney A, Charland P, et al. Optimizing the interprofessional workforce for centralized intake of patients with osteoarthritis and rheumatoid disease: case study. Hum Resour Health. 2015;13:41.
18. Lundon K, Shupak R, Schneider R, Herold Mcilroy J. Development and early evaluation of an inter-professional post-licensure education programme for extended practice roles in arthritis care. Physiother Can. 2011;63(1):94-103.

19. Lundon K, Kennedy C, Rozmovits L, et al. Evaluation of perceived collaborative behaviour amongst stakeholders and clinicians of a continuing education programme in arthritis care. $J$ Interprof Care. 2013;27(5):401-407.

20. Lundon K, Shupak R, Sunstrum-Mann L, Galet D, Schneider R. Leading change in the transformation of arthritis care: development of an interprofessional academic-clinical education training model. Healthc $Q$. 2008;11(3):62-68.

21. ACPAC Program Continuing Professional Development. Available from: https://acpacprogram.ca. Accessed May 14, 2018.

22. Farrer C, Abraham L, Jerome D, Hochman J, Gakhal N. Triage of rheumatology referrals facilitates wait time benchmarks. J Rheumatol. 2016;43(11):2064-2067.

23. Bombardier C, Brooks S, Bell M, Cesta A, Kendzerskaya T, Sweezie R, Widdifield J, Fullerton L, Ahluwalia V, Karasik A. The effect of triage assessments on identifying inflammatory arthritis and reducing Rheumatology wait times in Ontario [abstract]. Arthritis Rheumatol. 2016;68(suppl 10).

24. Mackay C, Davis AM, Mahomed N, Badley EM. Expanding roles in orthopaedic care: a comparison of physiotherapist and orthopaedic surgeon recommendations for triage. J Eval Clin Pract. 2009;15(1):178-183.

25. Stanhope J, Beaton K, Grimmer-Somers K, Morris J. The role of extended scope physiotherapists in managing patients with inflammatory arthropathies: a systematic review. Open Access Rheumatol. 2012;4:49-55.

26. Statistical Analytic System (SAS) [computer program]. Version 9.4. Cary, NC; 2016

27. Napier C, McCormack RG, Hunt MA, Brooks-Hill A. A physiotherapy triage service for orthopaedic surgery: an effective strategy for reducing wait times. Physiother Can. 2013;65(4):358-363.

28. Badley E, Veinot P, Tyas J, et al. 2006 Survey of Orthopaedic Surgeons in Ontario: ACREU Working Report. Toronto: University Health Network; March 2007.

29. Aiken AB, McColl MA. Diagnostic and treatment concordance between a physiotherapist and an orthopedic surgeon - a pilot study. J Interprof Care. 2008;22(3):253-261.

30. DiGiacomo M, Davidson PM, Zecchin R, Lamb K, Daly J. Caring for others, but not themselves: implications for health care interventions in women with cardiovascular disease. Nurs Res Pract. 2011;2011(3):376020.

31. Fullerton L, Brooks S, Sweezie R, Ahluwalia V, Bombardier C, Gagliardi A. Rheumatologist and extended role practitioner (ERP) perspectives on the implementation and impact of an allied health rheumatology triage initiative in Ontario rheumatology clinics. Poster presented at: Ontario Rheumatology Association Meeting; May 27-29, 2016; 2016; Toronto, ON.

32. Huscher D, Mittendorf T, von Hinüber U, et al. Evolution of cost structures in rheumatoid arthritis over the past decade. Ann Rheum Dis. 2015;74(4):738-745.

33. Widdifield J, Tu K, Carter Thorne J, et al. Patterns of care among patients referred to rheumatologists in Ontario, Canada. Arthritis Care Res. 2017;69(1):104-114.
Journal of Multidisciplinary Healthcare

\section{Publish your work in this journal}

The Journal of Multidisciplinary Healthcare is an international, peerreviewed open-access journal that aims to represent and publish research in healthcare areas delivered by practitioners of different disciplines. This includes studies and reviews conducted by multidisciplinary teams as well as research which evaluates the results or conduct of such teams or health
Dovepress

care processes in general. The journal covers a very wide range of areas and welcomes submissions from practitioners at all levels, from all over the world The manuscript management system is completely online and includes a very quick and fair peer-review system. Visit http://www.dovepress.com/ testimonials.php to read real quotes from published authors. 